\title{
MicroRNA-140-5p suppresses invasion and proliferation of glioma cells by targeting glutamate-ammonia ligase (GLUL)
}

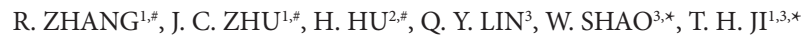 \\ ${ }^{1}$ Chinese People's Liberation Army No. 174 Clinical College, Anhui Medical University, Xiamen, China; ${ }^{2}$ Department of Pathology, Air Force \\ Hospital of Southern Theater Command, Guangzhou, China; ${ }^{3}$ Department of Pathology, Affiliated Chenggong Hospital, Xiamen University, \\ Xiamen, China
}

*Correspondence: vivishao@foxmail.com, skysea_ji@sina.com

${ }^{*}$ Contributed equally to this work.

Received May 14, 2019 / Accepted August 26, 2019

\begin{abstract}
Glutamine addiction is a major feature of glioma cells and plays an important role in its growth and proliferation. GLUL (glutamate-ammonia ligase), which catalyzes glutamate and ammonia to synthesize glutamine, plays a crucial role in tumor growth and proliferation. We attempt to determine a pathway that limits the growth of glioma by targeting GLUL and explore effective strategies blocking glutamine metabolism. We note that miRNAs mediate regulation of genes participating directly or indirectly in cancer cell metabolism. The regulatory roles of miRNAs on metabolic enzymes are widely discussed, however miRNAs regulation of glutamine metabolism by targeting GLUL in glioma has not yet been reported. Here, we examined both the expression and functions of GLUL in glioma cells. Findings indicated that the expression of GLUL was upregulated in high-grade compared to low-grade glioma cells. Knockdown of GLUL effectively inhibited proliferation, migration and invasion of glioma cells in vitro. Bioinformatics analyses, as well as dual-luciferase reporter assays, revealed that miR-140-5p bound to GLUL mRNA at the 3'-UTR location. Furthermore, the proliferation, migration and invasion of glioma cells were also repressed by miR-140-5p. Overall, these results showed that miR-140-5p exerted its inhibitory effects on proliferation, migration and invasion in glioma cells through downregulating GLUL. Thus, the miR-140-5p/GLUL axis may function as a potential target for glioma treatment.
\end{abstract}

Key words: glioma, miR-140-5p, GLUL, proliferation, metabolism

Glioma, a common brain malignant tumor, is associated with high morbidity, recurrence and mortality rates [1]. Gliomas have traditionally been diagnosed and treated based on the histopathological grades (I to IV) classified by the World Health Organization (WHO) [2]. Grade I-II gliomas are considered low-grade glioma (LGG), whereas grades III and IV, such as glioblastomas, are characterized as high-grade glioma (HGG). The degree of malignancy increases with grades [3]. The median overall survivals for LGG and HGG are 6-10 years or 12-15 months, respectively [4]. In HGG cases, less than 3-5\% of patients survive longer than 5 years after diagnosis $[4,5]$. Furthermore, over $50 \%$ of LGG cases transform into HGG within 5-10 years [6]. Despite advances in treatment, glioblastoma is essentially incurable. Further research is needed to fully explore the molecular mechanisms underlying the malignant transformation of gliomas.
Metabolic reprogramming is a major hallmark differentiating tumor cells from normal cells since it plays a crucial role in tumor initiation and progression [7]. In glioma, cell metabolism patterns change, due to an increased rate of glutamine consumption [8]. Glutamine is a rich and multifunctional nutrient in cancer cells that participates in redox homeostasis, energy formation, biomacromolecule synthesis and signal transduction [9]. Glutamate-ammonia ligase (GLUL) is a key enzyme in glutamine synthesis and has multiple functions in cell proliferation, signaling, glutamate and ammonia detoxification [10]. GLUL is highly expressed in multiple tumor types, including glioblastomas, breast cancer, hepatocellular carcinoma and prostate cancer [10-13]. In breast cancer, GLUL upregulation is associated with a poor prognosis. In addition, knockdown of GLUL significantly inhibits the proliferation of breast cancer cells [10]. Previous studies found that GLUL was highly expressed 
in glioblastoma and could promote the growth capacity of glioma cells $[11,14]$. Although these studies have elucidated the effects of GLUL on the biological functions of glioma, the mechanism regulating glutamine metabolism reprogramming in glioma remains to be further studied.

Increasing studies have shown that dysregulated miRNAs can regulate specific metabolic enzymes to accelerate cell metabolism in cancer [15]. A single miRNA has the ability to regulate several different genes, which can also be regulated by a variety of different miRNAs [16]. Thus, miRNAs are involved in complex regulatory networks that may influence all biological processes, such as cell growth, maturation, differentiation, energy generation, metabolism, invasion and migration $[17,18]$. In glucose metabolism, miR-143 decreases aerobic glycolysis via targeting Hexokinase 2 in some tumors, including esophageal squamous cell carcinoma, lung cancer and colorectal cancer [19]. Lactate dehydrogenase B is a target gene of miR-375, which affects tumor aggressiveness by regulating lactate metabolism in maxillary sinus and esophageal squamous cell carcinoma [20]. For glutamine metabolism, the research identified that miR-23a/b could enhance glutamine metabolism by regulating c-Myc [21]. However, the link between miRNAs and enzymes involved in glutamine metabolism requires further exploration.

Here, we revealed the relationship between GLUL expression and malignant grade in gliomas. GLUL was overexpressed in HGG cells, which was inversely related to the expression of miR-140-5p. Subsequently, the biological functions of GLUL were also explored in glioma cells. $\mathrm{miR}-140-5 \mathrm{p}$ was found to regulate the malignant progression of gliomas by directly targeting the GLUL mRNA.

\section{Materials and methods}

Cell culture. CHG5 and SHG44 cell lines were a gift from Professor Xiuwu Bian (The Third Military Medical University, China). The LN18 cell line was purchased from CCTCC (Chinese Typical Culture Preservation Center). U251 cells were obtained from ATCC (American Type Culture Collection). DMEM medium (Hyclone) was used to culture all cells used in this study.

Transfections. miR-140-5p mimics, miRNA negative controls (NC), GLUL siRNAs, GLUL negative control siRNAs (siRNA-NC), the pcDNA3.1-GLUL and control vector pcDNA3.1 were purchased from Gene Pharma (Shanghai, China). Lipofectamine 2000 reagent (ThermoFisher, USA) was used to transfect oligonucleotides or vectors into target cells. The transfection mixture media was replaced with 2 $\mathrm{ml}$ of fresh DMEM medium 6 hours after transfection. Cells were harvested for experiments $48 \mathrm{~h}$ after transfection.

RNA isolation and qRT-PCR. Tripure Isolation reagent (Roche, USA) was used to isolate total RNA from glioma cells. The Nanodrop 2000 spectrophotometer was used to determine the quality of RNA used in studies. For miR-140-5p and
GLUL analyses, the ReverTran Ace qPCR RT kit (Toyobo, Japan) was used to synthesize cDNA. Primers used in this study are listed in supplementary Table S1. The SYBR Green I Master mix (Roche, USA) and cobas z480 Fluorescent Quantitative PCR System (Roche, USA) were used to perform qPCR reactions.

Western blotting. RIPA lysis buffer was used to lyse cells. The BCA protein assay was performed to determine protein concentrations. Total protein $(20 \mu \mathrm{g})$ was separated using $10 \%$ SDS-PAGE and transferred to a PVDF membrane that was then blocked at room temperature with 5\% BSA for $1 \mathrm{~h}$. Blocked membranes were incubated with indicated primary antibodies overnight at $4{ }^{\circ} \mathrm{C}$ or for $2 \mathrm{~h}$ at room temperature and then incubated with HRP-conjugated secondary antibodies for $1 \mathrm{~h}$. Protein bands were detected using Pierce ${ }^{\mathrm{Tm}}$ ECL Western Blotting Substrate.

Cell proliferation. U251 and LN18 cells were plated in 96-well plates $\left(1 \times 10^{4}\right.$ cells/well) and incubated for 0, 24, 48 and $72 \mathrm{~h}$ at $37^{\circ} \mathrm{C}$ At the indicated times, $10 \mu \mathrm{l}$ of CCK- 8 reagent (Sigma) was added and cells were subsequently incubated at $37^{\circ} \mathrm{C}$ for $2 \mathrm{~h}$. The results were obtained using a Bio-Rad microplate spectrophotometer (Bio-Rad xMark, USA) at $450 \mathrm{~nm}$.

Transwell migration and invasion assays. Transwell chambers ( $8 \mu \mathrm{m}$ pores; Millipore, USA) were used to measure cell migration and invasion. In the Transwell migration assay, $300 \mu \mathrm{l}$ of serum free medium was used to suspend cells, which were then seeded into the upper chamber whereas $500 \mu \mathrm{l}$ of FBS contained DMEM was added into the lower chamber. After $24 \mathrm{~h}$, non-migrated cells were removed and remaining cells that migrated from the upper to the lower chamber were stained for $30 \mathrm{~min}$ using Giemsa. Stained cells were observed and counted using a microscope. Similar to what was described above, Matrigel (EMD Millipore, USA) coated transwell chambers were used to determine the invasion of cells in vitro.

Bioinformatics analyses. Potential miRNAs targeting the 3'-UTR of GLUL mRNA were predicted using TargetScan, miRanda and PITA miRNA databases.

Luciferase reporter assays. Human wild-type and mutant GLUL 3'-UTR sequences were designed and synthesized by GenePharma. The pmirGLO Dual-Luciferase miRNA Target Expression Vector (Promega, USA) was used to construct the wild-type GLUL 3'-UTR and mutant GLUL 3'-UTR luciferase reporter plasmids. Then, the constructed luciferase vectors were added together with miR-140-5p mimics or NCs into HEK293T cells in 96-well plates. After incubating for $48 \mathrm{~h}$, the activity of luciferase was measured. The firefly luciferase activities were normalized with the luciferase activity of Renilla.

Statistical analyses. GraphpadPrism7 was used to calculate and analyze experimental data. The Student's t-test was used to perform comparisons between two different groups. The ANOVA method was used to analyze data between multiple groups. All results were expressed as a mean \pm S.D. 


\section{Results}

Expression of GLUL in human glioma cell lines. Western blotting was performed to examine GLUL protein levels in different malignant glioma cell lines, including SHG44, CHG5, LN18 and U251 (Figure 1A). The SHG44 and CHG5 cell lines were obtained from LGG tissues, whereas the LN18 and U251 cell lines were derived from HGG tissues. The expression of GLUL in HGG cells was increased when compared to LGG cells (Figure 1A). qPCR was performed to investigate the mRNA expression of GLUL in glioma cell lines. Compared to LGG cells, GLUL mRNA expression levels were significantly increased in HGG cells (Figure 1B). These results suggested that higher expression of GLUL in gliomas might play a role in the biological functions of gliomas.

Knockdown of GLUL suppressed proliferation, migration and invasion of gliomas in vitro. Since GLUL is highly expressed in HGG cells, experiments repressing GLUL expression in LN18 and U251 cells were used to explore the biological functions of GLUL. The LN18 and U251 cells were transfected with GLUL-siRNA and knockdown efficiency was confirmed using qPCR and western blotting. Compared to the NC-siRNA group, protein and mRNA levels of GLUL in the LN18 and U251 GLUL-siRNA groups were decreased (Figures $2 \mathrm{~A}$ and B). These results indicated that GLUL-siRNA significantly reduced the expression of GLUL in the analyzed
A

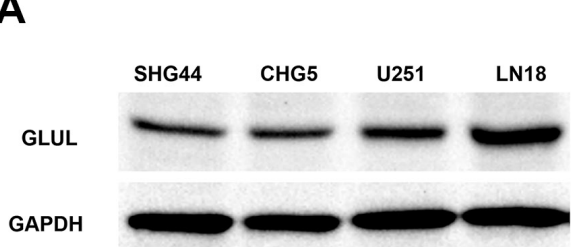

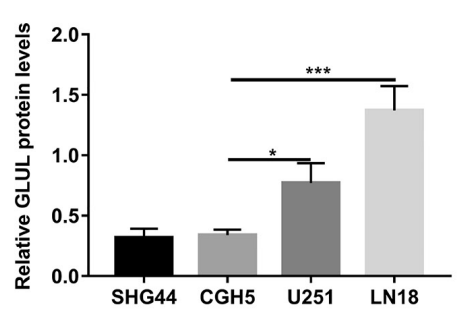

B

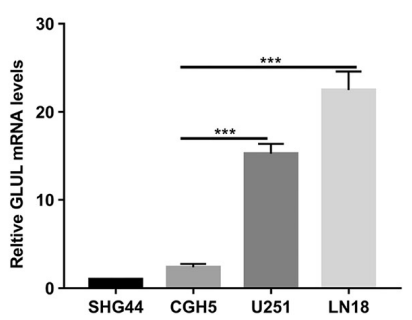

Figure 1. Expression of GLUL in human glioma cells. (A) The protein levels of GLUL in human glioma cells (SHG44, CHG5, U251 and LN18; ${ }^{\star}$ p $<0.05$; $\left.{ }^{* *} \mathrm{p}<0.01 ;{ }^{* *} \mathrm{p}<0.001\right)$. (B) The mRNA levels of GLUL in glioma cells as shown by $\mathrm{qPCR}$ analyses $\left({ }^{\star} \mathrm{p}<0.05\right)$. $\beta$-actin was used as an internal control.

A

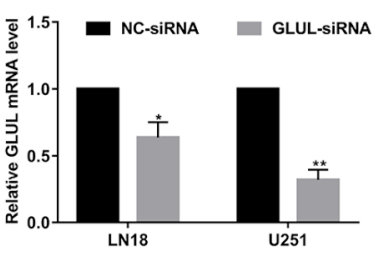

C
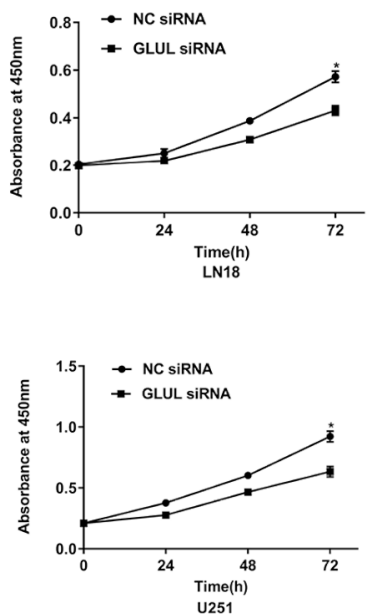

B
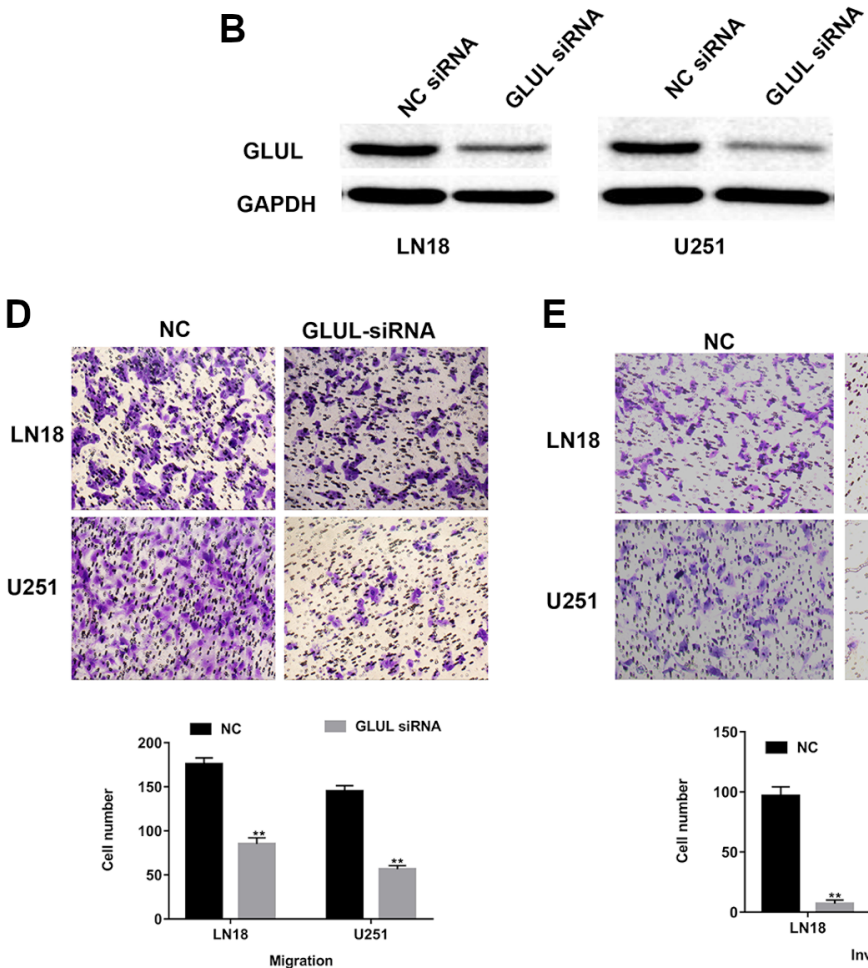

E
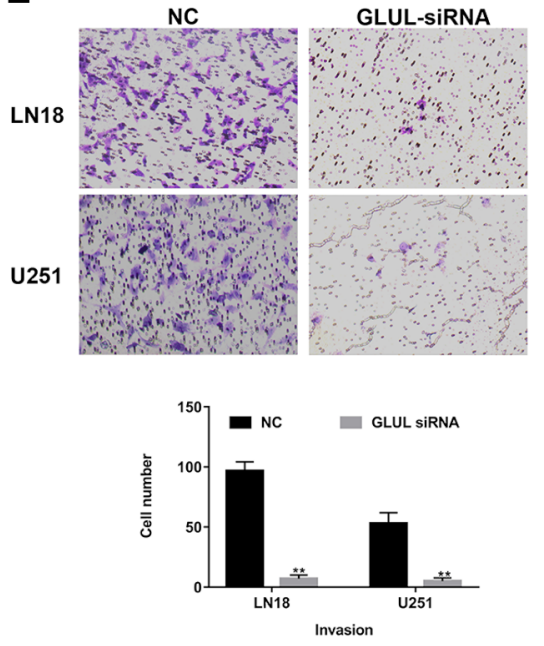

Figure 2. Knockdown of GLUL suppressed proliferation, migration and invasion of gliomas in vitro. (A and B) The expression of GLUL was determined by $\mathrm{qPCR}$ and western blotting in U251 and LN18 cells following transfection with GLUL siRNA/NC $\left({ }^{*} \mathrm{p}<0.05,{ }^{* *} \mathrm{p}<0.01\right)$. (C) CCK-8 assays on LN18 and U251 cells transfected with GLUL siRNA/NC $\left({ }^{*} \mathrm{p}<0.05\right)$. (D and E) Knockdown of GLUL inhibits the invasion (D) and migration (E) in LN18 and U251 cells $\left({ }^{* *} \mathbf{p}<0.01\right)$. 
A

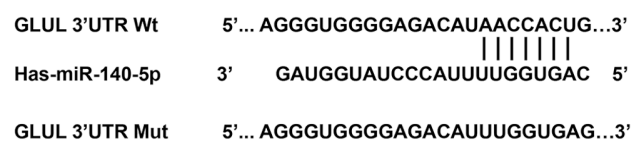

B

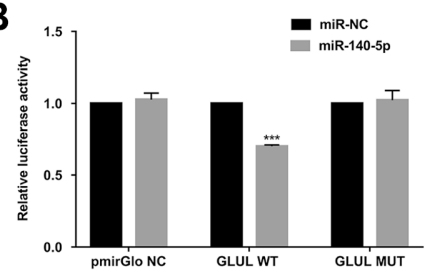

D

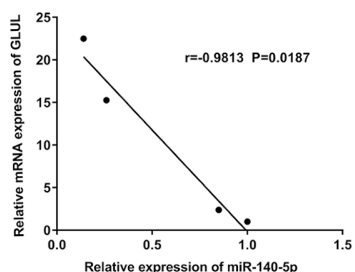

Figure 3. GLUL-3'UTR was targeted by miR-140-5p. Prediction of the binding sites of miRNA-140-5p in the GLUL 3'-UTR. (B) miR-140-5p mimics or NC together with pmirGLO-NC, pmirGLO-GLUL-3'UTR Wt or pmirGLO-GLUL-3'UTR Mut were transfected into HEK293T cells, then the luciferase activity was measured after 48 hours culture $\left({ }^{* *} \mathrm{p}<0.001\right)$. (C) miR-140-5p expression was detected in four glioma cell lines (SHG44, CHG5, U251 and LN8) using qPCR $\left({ }^{\star * *} \mathrm{p}<0.001\right)$. U6 was used as an internal control. (D) Spearman's correlation analysis of GLUL and miR-140-5p in glioma cell lines (SHG44, CHG5, U251 and LN18) $\left({ }^{*} \mathrm{p}<0.05\right)$.
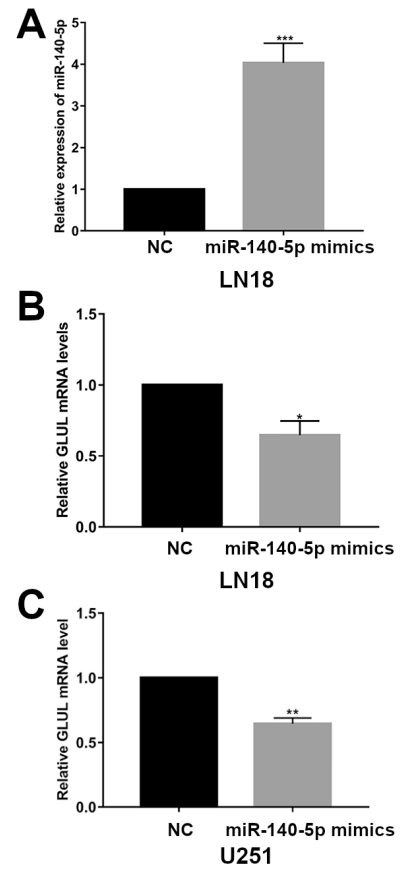

Figure 4. miR-140-5p suppressed GLUL expression. The efficiency of transfection was determined using $\mathrm{qPCR}$ method $\left({ }^{* * *} \mathrm{p}<0.001\right)$. The expression of GLUL in differentially treated cells was determined using qPCR (B) and western blot $(\mathrm{C})\left({ }^{*} \mathrm{p}<0.05,{ }^{* *} \mathrm{p}<0.01\right)$.

cell lines. To investigate the effects of GLUL on proliferation, a CCK-8 assay was used to show that knockdown of GLUL significantly suppressed LN18 and U251 cell proliferation activities (Figure 2C). Additionally, knockdown of GLUL significantly decreased the number of U251 and LN18 cells passing through the membrane compared to NC-siRNAtransfected group (Figures 2D and E). These observations indicated that GLUL participated in the malignant progression of glioma cells.

GLUL was one of miR-140-5p targets. According to the results obtained from several target analysis tools (TargetScan, miRanda and PicTar), a putative binding region between the GLUL gene sequence and miR-140-5p sequence was revealed, suggesting that GLUL 3'-UTR might be a target of miR-140-5p (Figure 3A). To further confirm this prediction, a dual-luciferase reporter gene assay was performed. miR-140-5p mimics or NC was transfected into HEK293T cells along with pmirGLO-NC, pmirGLO-GLUL-3'UTR or pmirGLO-GLUL-3'UTR Wt, respectively. As shown in Figure 3B, miR-140-5p mimics significantly reduced luciferase activity of the pmirGLO-GLUL-3'UTR Wt, while no obvious effect was observed on cells transfected with pmirGLO-GLUL-3'UTR Mut (Figure 3B), indicating that miR-140-5p directly interacted with the GLUL 3'-UTR. Next, using qPCR assays, we found miR-140-5p expression was dramatically lower in HGG cells compared to those in LGG cells (Figure 3C). Moreover, a negative correlation between the expression of GLUL and miR-140-5p was observed in glioma cells (Figure 3D). Our results indicated miR-140-5p might negatively regulate the expression of GLUL in gliomas.

miR-140-5p downregulated GLUL expression. To confirm whether the endogenous expression of GLUL was controlled by miR-140-5p, we overexpressed the miR-140-5p in LN18 and U251 cells by gene transfection method and then analyzed the expression of GLUL at the mRNA and protein levels. To confirm the efficiency of transfections, the expression levels of miR-140-5p were examined using qPCR. As shown in Figure 4A, the transfection of miR-140-5p mimics 
A

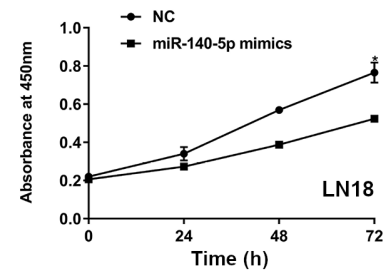

B

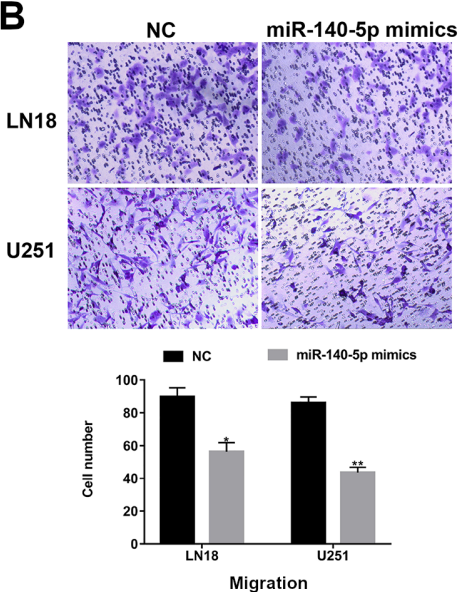

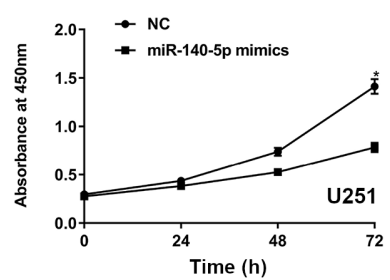

C

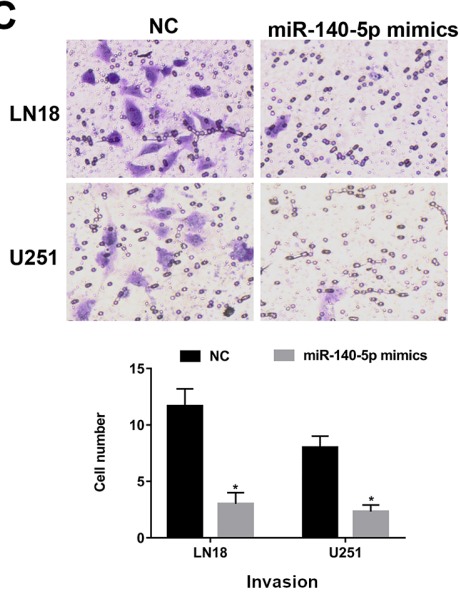

Figure 5. Proliferation, migration, and invasion were suppressed by miR-140-5p glioma cells. miR-140-5p mimics or NC was transfected in LN18 and U251 cells, then the proliferation (A), cell migration (B), and invasion (C) activity was detected with CCK-8 assay, transwell migration and invasion assay, respectively $\left({ }^{*} \mathrm{p}<0.05,{ }^{*} \mathrm{p}<0.05,{ }^{* *} \mathrm{p}<0.01\right.$, respectively).

successfully increased miR-140-5p expression in U251 and LN8 cells compared to controls (Figure 4A). A suppressive effect of miR-140-5p on the expression of GLUL in LN18 and U251 cells was also observed (Figures $4 \mathrm{~B}$ and $4 \mathrm{C}$ ).

Suppressive effects of miR-140-5p on proliferation, migration and invasion in glioma cells. miR-140-5p was found to bind to GLUL and downregulate GLUL expression in gliomas. Additional studies elucidating the mechanisms behind miR-140-5p in the proliferation, invasion and metastasis of glioma cells were needed. We observed that the proliferation, migration and invasion of LN18 and U251 were obviously impaired by the upregulation of miR-140-5p (Figure 5), which was similar to knocking down GLUL. These observations suggested miR-140-5p acted as a negative regulator of glioma phenotypes.

GLUL overexpression rescued the suppressive effects of miR-140-5p on proliferation and invasion in glioma cells. To further elucidate that miR-140-5p exerted tumor suppressive effect by downregulating GLUL, we performed a series of rescue experiments by overexpressing GLUL. After transfecting cells with a pcDNA3.1-GLUL expression vector, the expression of GLUL in miR-140-5p mimics-transfected cells was significantly restored (Figures 6A and 6B). Moreover, the suppressive effects of miR-140-5p on proliferation and invasion were also reversed by GLUL overexpression (Figures 6C-E). These results indicated that miR-140-5p suppressed invasion and proliferation of glioma cells by downregulating GLUL.

\section{Discussion}

Studies investigating cancer metabolism have shown that oncogenes and tumor suppressors could regulate cell metabolism. These studies also revealed that mutations in key metabolic enzymes are involved in the progression of cancers [22, 23]. Metabolic reprogramming is a major feature of tumors contributing to rapid cell survival and growth [7]. Glutamate/glutamine (Glu/Gln) homeostasis, a key mechanism for maintaining the normal cerebral function, is disrupted in glioma. The growth of glioma cells is strongly dependent on glutamine to provide increased biosynthesis and energy needs [8]. In early stages, research in our laboratory using $1 \mathrm{H}$ nuclear magnetic resonance (NMR)-based metabolic analysis found that glutamine metabolism patterns of glioma cells changed. Compared with low-grade glioma (LGG) cells, glutamine was significantly elevated in HGG cell metabolites [24]. GLUL is a key enzyme in the synthesis of glutamine and is crucial for the study of glutamine metabolism in the malignant transformation of gliomas.

In our present study, the expression of GLUL in HGG cells (LN18 and U251) was increased compared to GLUL expression in LGG cells (SHG44 and CHG5). Knockdown 
A

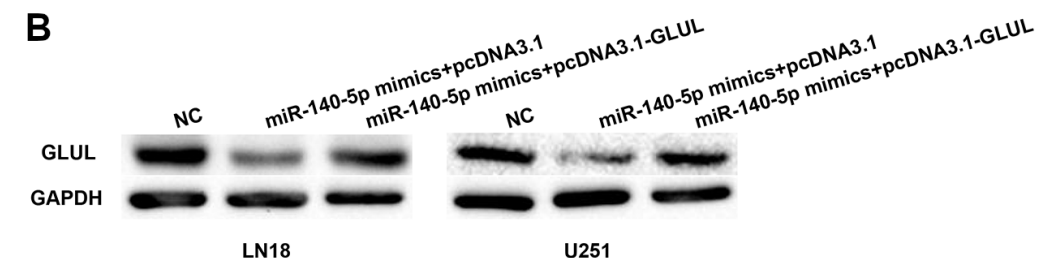

C \pm miR-140.5P mimicsspconNA3.1-GLLL
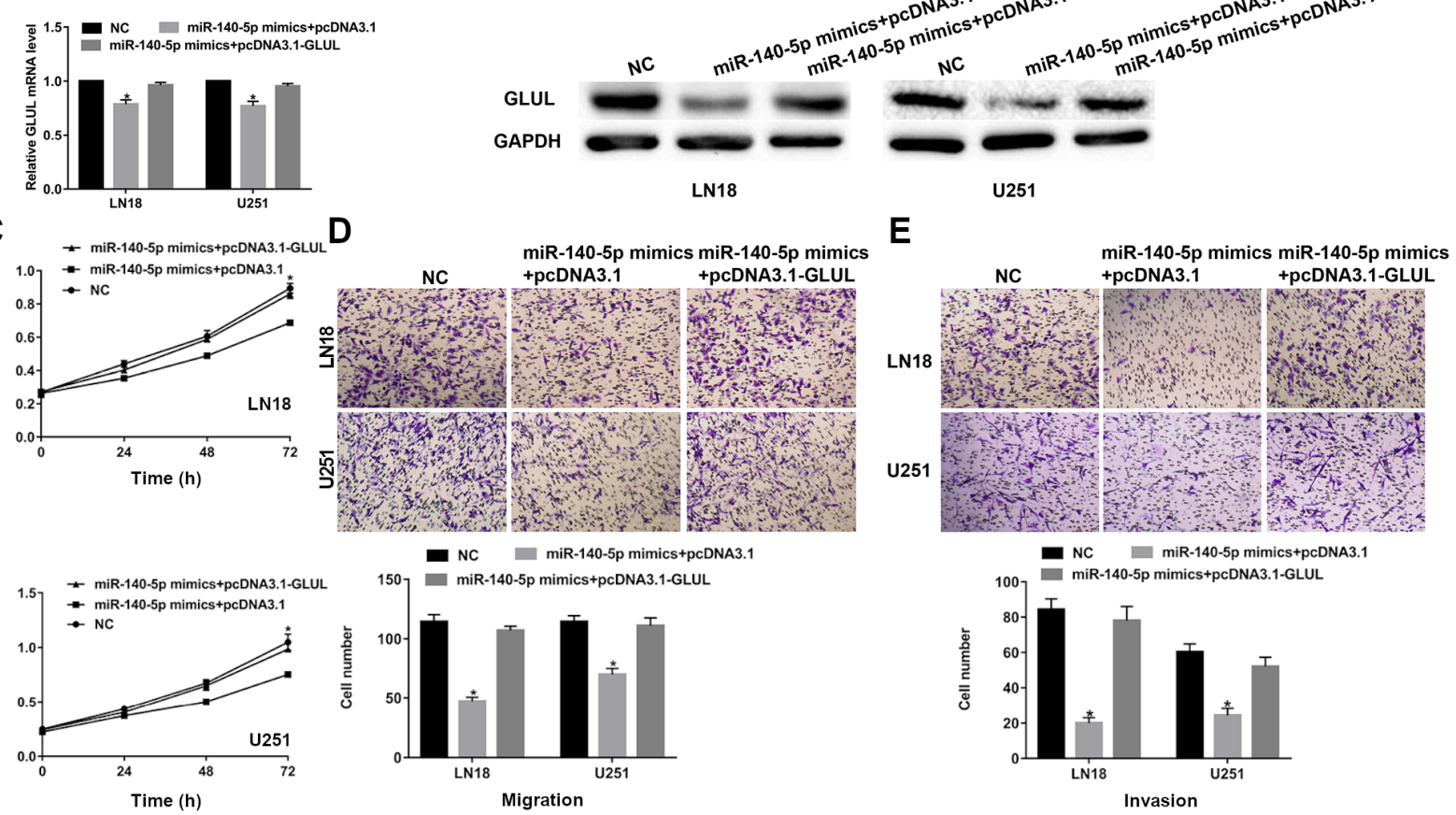

Figure 6. GLUL overexpression rescued the effect of miR-140-5p on glioma cells. (A and B) qPCR and western blotting analyses of GLUL expression in LN18 and U251 cells after co-transfection with miR-140-5p mimics and pcDNA3.1/pcDNA3.1-GLUL. GLUL overexpression rescued the suppressive effects of miR-140-5p in LN18 and U251 cells proliferation (C), migration (D), and invasion (E) $\left({ }^{*} \mathrm{p}<0.05\right)$.

of GLUL expression using siRNAs in LN18 and U251 cell lines indicated that GLUL repression significantly inhibited the proliferation, invasion and migration of glioma cells. This finding was supported by a previous study demonstrating that GLUL improves the growth capacity of glioblastomas [14]. Interestingly, a recent study found that the effect of GLUL on angiogenesis was greater than the synthesis of glutamine in pathological conditions [25]. These findings suggested that GLUL might play an essential role in the malignant transformation of gliomas. However, the specific mechanism of regulating GLUL expression in glioma is still unclear.

Based on TargetScan, miRanda and PITA miRNA databases, we predicted that GLUL might be a potential target of miR-140-5p. Numerous studies revealed miR-140-5p is involved in the occurrence and development of tumors [26-29]. For example, in hepatocellular carcinoma (HCC), miR-140-5p level correlated with patient prognosis and was significantly decreased compared to healthy controls. In addition, miR-140-5p also exerts its negative functions on cell metastasis and proliferation in HCC by targeting TGFBR1 and FGF9 [26]. A negative function of miR-140-5p on metastasis and proliferation was observed in gastric cancer through direct regulation of YES1 [27]. So far, several miR-140-5p target genes were identified, including VEGF-A, FGF9, TGFBR1, YES1, CEMIP and CADM3 [26-30]. However, the regulation mechanism between miR-140-5p and GLUL has not been elucidated in tumors.
Based on results obtained from luciferase assays, we concluded that miR-140-5p directly bound to GLUL mRNA at the 3'-UTR region. Additionally, compared to LGG cells (SHG44 and CHG5), HGG cells (LN18 and U251) expressed lower miR-140-5p levels. Moreover, between miR-140-5p and GLUL, a negative correlation was observed, indicating GLUL expression in glioma cells might be negatively regulated by miR-140-5p. This observation was also supported by the results that the expression of GLUL was markedly suppressed by miR-140-5p overexpression. Furthermore, the proliferation, invasion and migration capabilities of glioma cells were suppressed when miR-140-5p was overexpressed. These findings suggested that miR-140-5p could exert its tumor suppressive effects by downregulating the expression of GLUL.

In summary, we found that miR-140-5p overexpression and GLUL knockdown effectively inhibited glioma cell proliferation, invasion and migration. Meanwhile, we also observed that GLUL expression was commonly upregulated in HGG cells. Furthermore, we also identified GLUL as a novel target of miR-140-5p in glioma. All these findings indicated that miR-140-5p significantly inhibited the proliferation, invasion and migration of glioma cells by downregulating GLUL expression. These results contribute to the understanding of the molecular mechanisms of malignant progression of glioma at the metabolic level and provide an important theoretical basis to further explore the mecha- 
nism of glutamine metabolic reprogramming in glioma. The miR-140-5p/GLUL axis could possibly serve as a therapeutic target for glioma treatment.

Supplementary information is available in the online version of the paper.

Acknowledgements: This study was supported by Science and Technology Project of Xiamen (Grant No. 3502Z20174033, No. 3502Z20184064).

\section{References}

[1] CONSTANTIN A, ELKHALED A, JALBERT L, SRINIVASAN R, CHA $S$ et al. Identifying malignant transformations in recurrent low grade gliomas using high resolution magic angle spinning spectroscopy. Artif Intell Med 2012; 55: 6170. https://doi.org/10.1016/j.artmed.2012.01.002

[2] WEN PY, HUSE JT. 2016 World Health Organization Classification of Central Nervous System Tumors. Continuum (Minneap Minn) 2017; 23: 1531-1547. https://doi. org/10.1212/CON.0000000000000536

[3] KUMTHEKAR P, RAIZER J, SINGH S. Low-Grade Glioma. Cancer Treat Res 2015; 163: 75-87. https://doi. org/10.1007/978-3-319-12048-5_5

[4] CASTRO MG, CANDOLFI M, KROEGER KM, KING GD, CURTIN JF et al. Gene Therapy and Targeted Toxins for Glioma. Curr Gene Ther 2011; 11: 155-180. https://doi. org/10.2174/156652311795684722

[5] PARSONS DW, JONES S, ZHANG X, LIN JC, LEARY RJ et al. An integrated genomic analysis of human glioblastoma multiforme. Science. 2008; 321: 1807-1812. https://doi. org/10.1126/science. 1164382

[6] MAHER EA, FURNARI FB, BACHOO RM, ROWITCH $\mathrm{DH}$, LOUIS DM et al. Malignant glioma: genetics and biology of a grave matte. Genes Dev 2001; 15: 1311-1333. https:// doi.org/10.1101/gad.891601

[7] VANDER HEIDEN MG, DEBERARDINIS RJ. Understanding the intersections between metabolism and cancer biology. Cell 2017; 168: 657-669. https://doi.org/10.1016/j. cell.2016.12.039

[8] MÁRQUEZ J, ALONSO FJ, MATÉS JM, SEGURA JA, MARTÍN-RUFIÁN M et al. Glutamine Addiction In Gliomas. Neurochem Res 2017; 42: 1735-1746. https://doi. org/10.1007/s11064-017-2212-1

[9] HENSLEY CT, WASTI AT, DEBERARDINIS RJ. Glutamine and cancer: cell biology, physiology, and clinical opportunities. J Clin Invest 2013; 123: 3678-3684. https://doi. org/10.1172/JCI69600

[10] WANG Y, FAN S, LU J, ZHANG Z, WU D et al. GLUL Promotes Cell Proliferation in Breast Cancer. J Cell Biochem 2017; 118: 2018-2025. https://doi.org/10.1002/jcb.25775

[11] MARIN-VALENCIA I, YANG C, MASHIMO T, CHO S, BAEK $\mathrm{H}$ et al. Analysis of tumor metabolism reveals mitochondrial glucose oxidation in genetically diverse human glioblastomas in the mouse brain in vivo. Cell Metab 2012 6; 15: 827-837. https://doi.org/10.1016/j.cmet.2012.05.001
[12] SHI X, ZHANG X, YI C, LIU Y, HE Q. $\left[{ }^{13} \mathrm{~N}\right]$ Ammonia positron emission tomographic/computed tomographic imaging targeting glutamine synthetase expression in prostate cancer. Mol Imaging 2014; 13. https://doi. org/10.2310/7290.2014.00048

[13] LONG J, LANG ZW, WANG HG, WANG TL, WANG BE et al. Glutamine synthetase as an early marker for hepatocellular carcinoma based on proteomic analysis of resected small hepatocellular carcinomas. Hepatobiliary Pancreat Dis Int 2010; 9: 296-305.

[14] TARDITO S, OUDIN A, AHMED SU, FACK F, KEUNEN $\mathrm{O}$ et al. Glutamine synthetase activity fuels nucleotide biosynthesis and supports growth of glutamine-restricted glioblastoma. Nat Cell Biol 2015; 17: 1556-1568. https://doi. org/10.1038/ncb3272

[15] HATZIAPOSTOLOU M, POLYTARCHOU C, ILIOPOULOS D. miRNAs link metabolic reprogramming to oncogenesis. Trends Endocrinol Metab 2013; 24: 361-373. https:// doi.org/10.1016/j.tem.2013.03.002

[16] AMBROS V. The functions of animal microRNAs. Nature 2004 16; 431: 350-355. https://doi.org/10.1038/nature02871

[17] BARTEL DP. MicroRNAs: Genomics, biogenesis, mechanism, and function. Cell 2004; 116: 281-297. https://doi. org/10.1016/s0092-8674(04)00045-5

[18] LAI EC. microRNAs: Runts of the Genome Assert Themselves. Curr Biol 2003; 13: R925-R936. https://doi. org/10.1016/j.cub.2003.11.017

[19] GREGERSEN LH, JACOBSEN A, FRANKEL LB, WEN JY, KROGH A et al. MicroRNA-143 down-regulates Hexokinase 2 in colon cancer cells. BMC Cancer 2012; 12: 232. https://doi.org/10.1186/1471-2407-12-232

[20] KINOSHITA T, NOHATA N, YOSHINO H, HANAZAWA T, KIKKAWA $\mathrm{N}$ et al. Tumor suppressive microRNA-375 regulates lactate dehydrogenase $\mathrm{B}$ in maxillary sinus squamous cell carcinoma. Int J Oncol 2012; 40: 185-193. https:// doi.org/10.3892/ijo.2011.1196

[21] GAO P, TCHERNYSHYOV I, CHANG TC, LEE YS, KITA $\mathrm{K}$ et al. c-Myc suppression of miR-23a/b enhances mitochondrial glutaminase expression and glutamine metabolism. Nature 2009; 458: 762-765. https://doi.org/10.1038/ nature 07823

[22] DANG CV. Links between metabolism and cancer. Genes Dev 2012; 26: 877-890. https://doi.org/10.1101/ gad.189365.112

[23] MULLEN AR, DEBERARDINIS RJ. Genetically-defined metabolic reprogramming in cancer. Trends Endocrinol Metab 2012; 23: 552-559. https://doi.org/10.1016/j. tem.2012.06.009

[24] SHAO W, GU J, HUANG C, LIU D, HUANG H et al. Malignancy-associated metabolic profiling of human glioma cell lines using 1H NMR spectroscopy. Mol Cancer 2014; 13: 197. https://doi.org/10.1186/1476-4598-13-197

[25] EELEN G, DUBOIS C, CANTELMO AR, GOVEIA J, BRUNING $U$ et al. Role of glutamine synthetase in angiogenesis beyond glutamine synthesis. Nature 2018; 561: 6369. https://doi.org/10.1038/s41586-018-0466-7 
[26] YANG H, FANG F, CHANG R, YANG L. MicroRNA-140-5p suppresses tumor growth and metastasis by targeting transforming growth factor beta receptor 1 and fibroblast growth factor 9 in hepatocellular carcinoma. Hepatology 2013; 58: 205-217. https://doi.org/10.1002/hep.26315

[27] FANG Z, YIN S, SUN R, ZHANG S, FU M et al. miR-140-5p suppresses the proliferation, migration and invasion of gastric cancer by regulating YES1. Mol Cancer 2017; 16: 139. https://doi.org/10.1186/s12943-017-0708-6

[28] LU Y, QIN T, LI J, WANG L, ZHANG Q et al. MicroRNA140-5p inhibits invasion and angiogenesis through targeting VEGF-A in breast cancer. Cancer Gene Ther 2017; 24: 386-392. https://doi.org/10.1038/cgt.2017.30
[29] LI W, HE F. Monocyte to macrophage differentiation-associated (MMD) targeted by miR-140-5p regulates tumor growth in non-small cell lung cancer. Biochem Biophys Res Commun 2014; 450: 844-850. https://doi.org/10.1016/j. bbrc.2014.06.075

[30] MIAO X, WANG Z, CHEN B, CHEN Y, WANG X et al. miR-140-5p suppresses retinoblastoma cell proliferation, migration, and invasion by targeting CEMIP and CADM3. Cell Mol Biol (Noisy-le-grand) 2018; 64: 42-47. 


\section{MicroRNA-140-5p suppresses invasion and proliferation of glioma cells by targeting glutamate-ammonia ligase (GLUL)}

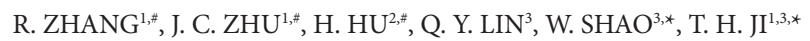

\section{Supplementary Information}

Suppl. Table S1. qPCR primers used in this study.

\begin{tabular}{lll}
\hline Target & Primer & Sequence \\
\hline miR-140-5p & F Primer & CGCCTACAGTGGTTTTACCCT \\
& R Primer & TATGGTTTTGACGACTGTGTGAT \\
U6 & F Primer & CAGCACATATACTAAAATTGGAACG \\
& R Primer & ACGAATTTGCGTGTCATCC \\
GLUL & F Primer & TCATCTTGCATCGTGTGTGTG \\
& R Primer & CTTCAGACCATTCTCCTCCCG \\
\multirow{3}{*}{-actin } & F Primer & TCCATCATGAAGTGTGACGT \\
& R Primer & TACTCCTGCTTGCTGATCCAC \\
\hline
\end{tabular}

